\title{
Clinical Trial Subject Enrollment Log
}

National Cancer Institute

\section{Source}

National Cancer Institute. Clinical Trial Subject Enrollment Log. NCI Thesaurus. Code C115537.

Records identifying all subjects enrolled in a clinical trial. 